\title{
Comparison between "In-bore" MRI guided prostate biopsy and standard ultrasound guided biopsy in the patient with suspicious prostate cancer: Preliminary results
}

\author{
Daniele D'Agostino ${ }^{1}$, Federico Mineo Bianchi ${ }^{2}$, Daniele Romagnoli ${ }^{1}$, Paolo Corsi ${ }^{1}$, Marco Giampaoli ${ }^{1}$, \\ Riccardo Schiavina ${ }^{2}$, Eugenio Brunocilla ${ }^{2}$, Walter Artibani ${ }^{1}$, Angelo Porreca ${ }^{1}$ \\ ${ }^{1}$ Department of Robotic Urological Surgery, Abano Terme Hospital, Abano Terme, Italy; \\ ${ }^{2}$ Department of Urology, University of Bologna, Bologna, Italy.
}

\begin{abstract}
Summary Objectives: To evaluate the detection rate of prostate cancer $(\mathrm{PCa})$ in patients who underwent to "in bore" Magnetic Resonance Imaging -guided prostate (MRI-GB) biopsy compared to the standard transrectal ultrasound guided prostate biopsy (TRUS-GB).

Materials and methods: Between January 2017 and March 2015 a cohort of 39 consecutive patients was prospectively enrolled. All the patients underwent an "in-bore" guided MRI prostatic biopsy and subsequently ultrasound-guided standard prostate biopsy. Results: Median age of patients was 65.5 years ( $S D \pm 6.6$ ), median total PSA serum level was $6.6 \mathrm{ng} / \mathrm{ml}(S D \pm 4.1)$, median prostate total volume was $51.1 \mathrm{cc}(S \mathrm{SD} \pm 26.7)$. Thirty of 39 (76.9\%) were biopsy-naïve patients while 7/39 (17.9\%) had at least one previous negative random TRUS-GB; 2/39 (5.1\%) patients were already diagnosed as PCa and were on active surveillance. In 18/39 (53.8\%) men Pca was diagnosed; as regards the MRI-GB results related to the PI-RADS score, biopsies of PIRADS 3 lesions were positive in 5/18 cases (27.8\%), while the number of positive cases of PI-RADS 4 and 5 lesions was 7/11 $(63.6 \%)$ and $6 / 10(60 \%)$ respectively. At the histological examination, $4 / 39(10.3 \%)$ had a PCa ISUP grade group $1,11 / 39(28.2 \%)$ had a ISUP 2, 6/39(15.4\%) had a ISUP grade group 3 and 2/39 (5.1\%) had a ISUP 4-5.

Conclusions: MRI-GB represents a promising technique that may offer some of advantages compared to standard systematic TRUSGB. Our preliminary experience in MRI-GB resulted safe and feasible and represents a viable procedure for the diagnosis and characterization of $\mathrm{PC}$.
\end{abstract}

KEY WORDS: Prostate cancer; MRI guided biopsy; Ultrasound guided biopsy.

Submitted 27 March 2019; Accepted 29 April 2019

\section{INTRODUCTION}

Prostate Cancer (PCa) represents the most common neoplasm diagnosed in men and the second cause of death after lung cancer (1). Digital rectal examination, serum PSA assay and ultrasound-guided biopsy (TRUS-GB) are common methods for prostate cancer diagnosis (2) although not devoid of some limitations and questionable validity. In fact, during prostate ultrasound, the yield of cancer detection remains very low, ranging between 20 and 30\% for patients with a serum PSA value between 2 and $4 \mathrm{ng} / \mathrm{ml}(3,4)$; moreover, in a non-negligible per- centage of cases (25-30\%), PCa may arise from the anterior part of the gland (anterior horn of peripheral gland, central/transitional zones and fibromuscular stroma). For these reasons, "random" TRUS-GB has a false negative rate of up to $40-50 \%(5,6)$ and clinically significant tumors can often be undetected, especially in larger glands. A higher number of cores can improve the rate of tumors identified but, at the same time, increases the risk of diagnosis of indolent diseases: an aggressive management of these overdiagnosed cases will inevitably lead to an overtreatment, thereby negatively affecting the patients' quality of life. Magnetic Resonance Imaging (MRI) is a tool of growing importance in $\mathrm{PCa}$ diagnosis; furthermore, with the introduction of multiparametric MRI (mpMRI) the accuracy for characterization of prostate lesion is significantly improved. mpMRI, performed before the biopsy, can improve the detection of occult tumors in the areas of prostate generally undersampled during random TRUS-GB. A growing body of evidences suggest that an accurate tumor identification and sampling can improve risk classification and should reduce false-negative rates and the necessity of repeat biopsies both in biopsy-naive patients and in patients with prior negative-biopsy (710). However, the increasingly widespread use of the mpMRI for the detection of clinically significant PCa has involved the need for targeted biopsy reducing the sampling in non-suspicious areas. The techniques for targeted biopsy include visual estimation TRUS-GB, software co-registered MRI ultrasound fusion and in-bore MRIguided biopsy (MRI-GB); the latter provides the great advantage of direct visualization of the targeted lesion with the same system. Thus, the aim this study was to evaluate the detection rate of prostate cancer in patients underwent to "in bore" MRI-guided prostate biopsy compared to the standard ultrasound guided prostate biopsy; for secondary endpoint we evaluated the morbility and the complications of both procedures.

\section{Materials AND METHOdS}

Study methodology and population

Between January 2017 and March 2015 a cohort of 39 
consecutive patients was prospectively enrolled. The study was conducted with the approval of the institutional review board and all the procedures and reporting were in accordance with the Helsinki protocol. Patients were informed and a subsequent written consent was acquired. All patients had a suspicion for $\mathrm{PCa}$ - raised Prostate Specific Antigen (PSA) serum level and/or abnormal digital rectal examination (DRE) - and showed at the mp-MRI previously conducted in our institution at least one suspicious area where the presence of a clinically significant PCa was defined equivocal/ikely/highly likely, corresponding to a PI-RADS (Prostate Imaging Reporting and Data System) score $\geq 3$ according to the European Society of Urogenital Radiology (ESUR) guidelines (17). Patients with any usual contraindications for MRI (eg. metallic implants and/or cardiac pacemakers) were excluded from the study. All patients underwent a "in-bore" guided MRI prostatic biopsy and subsequently ultrasound-guided standard prostate biopsy; the urologist who performed the ultrasound-guided prostatic biopsy was always the same and was not aware of the result of mpMRI.

\section{MRI examination and analysis}

All the MRI examinations were performed with a 32 channels 1.5 T whole body scanner (Achieva XR; Philips Medical Systems, Best, Netherlands) with a 32-channels phasedarray surface coil without endorectal coil. After local threeplane acquisition, required for the correct positioning of the sequences, the morphological and functional studies were carried out. Morphological study of the prostate gland were obtained with Turbo Spin Echo (TSE) T2weighted sequences (TE $100 \mathrm{msec}$, TR $4074 \mathrm{msec}$, Slice Thickness $3 \mathrm{~mm}$, Slice Spacing $0.3 \mathrm{~mm}$, Field of View - FOV $180 \times 180 \mathrm{~mm}$ and matrix size $276 \times 205)$ in the sagittal, axial and coronal planes, including seminal vesicles and the entire prostate gland. For the functional study, DWI, DCE-MRI and MRS acquisition were performed. The DWI acquisition was carried out in the axial plane, using a single-shot echo-planar imaging (SSEPI) sequence, with three $\mathrm{b}$-values $\left(0,600\right.$ and $\left.1500 \mathrm{~s} / \mathrm{mm}^{2}\right)$, slice thickness of 3 $\mathrm{mm}$, FOV $180 \times 180 \mathrm{~mm}$ and matrix size $80 \times 71$. The DCE-MRI was obtained using three-dimensional (3D) T1W High Resolution Isotropic Volume Examination (THRIVE) sequence during the intravenous injection of a contrast bolus of 0,1 mmol per kilogram of body weight of Meglumine gadobenate (Multihance, Bracco Diagnostics, Milan, Italy), at flow rate of 3,5 ml/sec followed by $15 \mathrm{ml}$ of saline solution.

\section{Conduct of the "in-bore" MRI-guided biopsy}

The biopsies were performed on a different day from the diagnostic mpMRI study (within 2-4 weeks) by a single urologist. All patients received oral antibiotic prophylactic therapy 2 days before the maneuver; biopsies were performed transrectally with the patient prone on a 1.5 T MR scanner (Achieva XR; Philips Medical Systems, Best, Netherlands) using a 18-G automatic core-needle, a titanium double-shot biopsy gun, a Gadolinium-filled needle-guide, a non-magnetic portable biopsy device (DynaTRIM, Invivo, Gainesville, FL) and a dedicated software package for device tracking and target localization (DynaCAD, Invivo, Gainesville, FL). Before biopsy, DRE was performed in order to evaluate any potential anatomic or pathologic condition that could hinder transrectal biopsy and to approximate the position of the gland. The needle sleeve is also a marker for software localization; after the sleeve was assembled on the arm of the biopsy device, fixed on the tabletop of the magnet, and inserted into the rectum, the patient was positioned into the scanner. T2w images in the axial and sagittal direction were obtained for visualization of the prostate and identification of the suspicious lesion; simultaneously, the Gadolinium-filled needle guide was properly identified and marked for subsequent track calibration: oblique axial $\mathrm{T} 2 \mathrm{w}$ images were aligned with the needle guide in order to allow software registration showing three-dimensional adjustments required to align the track of the biopsy needle through the needle guide and the target lesion. After manual calibration adjustments on the arm of the biopsy device attached to the needle guide, sagittal T2w images in parallel with the long axis were obtained in order to confirm the correct position and the proper direction of the needle guide to the target; reconfirmation of the needle track were repeated until proper alignment was obtained. Once the patient was taken from the scanner, biopsy was performed with a median of 2 (range 1-2) cores taken from each target lesion. If targeting was not certain, due to lesion size or subjective judgement of the operator, subsequent axial and sagittal $\mathrm{T} 2 \mathrm{w}$ images with the needle in place were obtained to detect needle position and be able to make adjustments for the next core. Total table time was appreciatively 45 to 70 minutes per patient. After the procedure, patients were observed for 1 hour and were re-evaluated by outpatient visit after 7-10 days in order to record any potential complication. Specimens were processed by routine hystopathological fixation with formalin solution and evaluated by a single dedicated uropathologist with 20 years of experience.

\section{Conduct of ultrasound guided prostate biopsy}

The standard biopsy was performed by transrectal approach; the examination was tipically 12 cores collected in a sextant template of biopsies from the medial and lateral area of the apical, mid and the base of prostate, on the left and right lobe. During the TRUS guided biopsy the mpMRI data target was not available. Total table time was appreciatively 15 to 30 minutes per patient. After the procedure, patients were observed for 1 hour and were re-evaluated by outpatient visit after 7-10 days in order to record any potential complication. antibiotic prophylaxis was continued by patients for seven days after the procedure. Specimens were processed by routine hystopathological fixation with formalin solution and evaluated by a single dedicated uro-pathologist with 20 years of experience.

\section{Statistical analysis}

Simple descriptive statistical techniques were used to analyze data, in particular median with interquartile range (IQR) and mean \pm standard deviation (SD) were used to report continuous variables, whereas frequencies with percentages were used to describe categorical ones. Oneway ANOVA and Kruskal-Wallis k-samples were used to 
compare means and medians between three groups. Pearson's Chi-Square test and Mc-Nemar test were used to compare the association between clinical and pathologic variables. Two-tailed $\mathrm{P}$ values less than 0.05 were considered statistically significant. Statistical analyses were conducted using SPSS ${ }^{\circledR}$ version 21.0 for Macintosh ${ }^{\circledR}$ (IBM Corp, Armonk, NY).

\section{Results}

Patient characteristics: Median age of patients was 65.5 years $(S D \pm 6.6)$, median total PSA serum level was 6.6 $\mathrm{ng} / \mathrm{ml}(\mathrm{SD} \pm 4.1)$, median prostate total volume was $51.1 \mathrm{cc}(\mathrm{SD} \pm 26.7)$. Thirty of 39 (76.9\%) were biopsynaive patients while $7 / 39$ (17.9\%) had at least one previous negative random TRUS-GB; 2/39 (5.1\%) patients were already diagnosed $\mathrm{pCa}$ on active surveillance. Clinical, radiological and pathological characteristics of the entire population of the study are listed in Table 1.

Detection rate All patient enrolled in the study have been subjected both the MRI-GB and the TRUS-GB. The majority of suspected lesions identified with mpMRI were located in the posterior zone of the prostate (69\% of cases).

The mean number of bioptic cores taken from patient was 1 in MRI-GB series (range 1-2) and 12 in TRUS-GB (range 12-14).As reported in table 5, about overall detection rate (DR) of patients who underwent "in-bore" MRIGB and TRUS-GB, in 23/39 (59\%) men PCa was diagnosed; as regards the MRIGB results related to the PI-RADS score, biopsies of PI-RADS 3 lesions were positive in $5 / 18$ cases $(27.8 \%$ ), while the number of positive cases of PI-RADS 4 and 5 lesions was $7 / 11$ (63.6\%) and 6/10 (60\%) respectively. Analyzing the results of MRI-GB, globally 17/39 (43.5\%) patients presented a clinically significant PCa (ISUP group $\geq 2$ ), while the DR for clinically significant PCa in TRUS-GB was $15 / 39$ patients (38.4\%). In the series of "biopsy naïve" patient we observed 14/30 (46.7\%) cases of PCa detected by MRI-GB and 13/30 (43.3\%) cases in the series of TRUS-GB. In patients with previous negative biopsies we observed PCa in 2/7 (28.6\%) cases both in MRI-GB and TRUS-GB. In the "active surveillance" group we detected 2/2 (100\%) men with PCa in MRI-GB series and $1 / 2(50 \%)$ in TRUS-GB
Table 1.

Clinical and radiologic features of patients who underwent "in-bore" biopsy.

\begin{tabular}{|c|c|c|c|c|c|}
\hline & $\begin{array}{l}\text { Overall } \\
(n=39)\end{array}$ & $\begin{array}{l}\text { Biopsy } \\
\text { näive } \\
(n=30)\end{array}$ & $\begin{array}{c}\text { Previous } \\
\text { negative biopsy } \\
(n=7)\end{array}$ & $\begin{array}{c}\text { Active } \\
\text { surveillance } \\
(n=2)\end{array}$ & $\begin{array}{c}\mathbf{p} \\
\text { value }\end{array}$ \\
\hline $\begin{array}{l}\text { Age, years } \\
\text { Mean } \pm \text { SD }\end{array}$ & $65.5 \pm 6.6$ & $65.3 \pm 6.8$ & $66.6 \pm 7.1$ & $63.5 \pm 2.1$ & 0.8 \\
\hline $\begin{array}{l}\text { PSA, ng/ml } \\
\text { Mean } \pm \text { SD }\end{array}$ & $6.6 \pm 4.1$ & $11.7 \pm 4.9$ & $10.9 \pm 7.4$ & $6.2 \pm 1$ & 0.007 \\
\hline $\begin{array}{l}\text { PSA density, ng/ml/cc } \\
\text { Mean } \pm \text { SD }\end{array}$ & $0.14 \pm 0.09$ & $0.14 \pm 0.09$ & $0.15 \pm 0.05$ & $0.13 \pm 0.04$ & 0.95 \\
\hline $\begin{array}{l}\text { Prostate volume, cc } \\
\text { Mean } \pm \text { SD }\end{array}$ & $51.1 \pm 26.7$ & $46.7 \pm 22.2$ & $70.4 \pm 38.1$ & $52 \pm 8.5$ & 0.1 \\
\hline $\begin{array}{l}\text { Diameter mpMRI area, mm } \\
\text { Mean } \pm \mathrm{SD}\end{array}$ & $11.9 \pm 5.1$ & $11.7 \pm 4.9$ & $13.1 \pm 6.5$ & $10 \pm 5.7$ & 0.7 \\
\hline \multicolumn{6}{|l|}{ Area location mpMRI+, n (\%) } \\
\hline $\begin{array}{l}\text { Posterior } \\
\text { Anterior }\end{array}$ & $\begin{array}{l}27(69.2) \\
12(30.8)\end{array}$ & $\begin{array}{l}21(70) \\
9(30)\end{array}$ & $\begin{array}{l}4(57.1) \\
3(42.9)\end{array}$ & $\begin{array}{c}2(100) \\
0(0)\end{array}$ & 0.5 \\
\hline \multicolumn{6}{|l|}{ PI-RADS-v2, n (\%) } \\
\hline $\begin{array}{l}3 / 5 \\
4 / 5 \\
5 / 5\end{array}$ & $\begin{array}{l}18(46.2) \\
11(28.2) \\
10(25.6)\end{array}$ & $\begin{array}{l}14(46.7) \\
8(26.7) \\
8(26.7)\end{array}$ & $\begin{array}{l}2(28.6) \\
3(42.9) \\
2(28.6)\end{array}$ & $\begin{array}{c}2(100) \\
0(0) \\
0(0)\end{array}$ & 0.5 \\
\hline
\end{tabular}

Table 2.

Overall and stratified according to clinical status "in-bore" results.

\begin{tabular}{|lccccc|}
\hline & Overall & $\begin{array}{c}\text { Biopsy } \\
\text { näive }\end{array}$ & $\begin{array}{c}\text { Previous } \\
\text { negative biopsy }\end{array}$ & $\begin{array}{c}\text { Active } \\
\text { surveillance }\end{array}$ & $\begin{array}{c}\mathbf{p} \\
\text { value }\end{array}$ \\
\hline Detection rate PCa, $\mathrm{n}(\%)$ & $18 / 39(53.8)$ & $14 / 30(46.7)$ & $2 / 7(28.6)$ & $2 / 2(100)$ & 0.2 \\
\hline Detection rate csPCa, $\mathrm{n}(\%)$ & $17 / 39(43.5)$ & $14 / 30(46.6)$ & $2 / 7(28.6)$ & $1 / 2(50)$ & 0.1 \\
\hline csPCa/overall PCa rate, $\%$ & $17 / 18(94.4)$ & $14 / 14(100)$ & $2 / 7(28.6)$ & $1 / 2(50)$ & 0.2 \\
\hline ISUP grade group, $\mathrm{n}(\%)$ & $21(53.8)$ & $16(53.3)$ & $5(71.4)$ & $0(0)$ & 0.1 \\
Negative & $1(2.5)$ & $0(0)$ & $0(0)$ & $1(50)$ & \\
1 & $10(25.6)$ & $10(33.3)$ & $0(0)$ & $0(0)$ & \\
2 & $4(10.3)$ & $2(6.6)$ & $1(14.2)$ & $1(50)$ & \\
3 & $3(7.6)$ & $2(6.6)$ & $1(14.2)$ & $0(0)$ & \\
$4-5$ & & & & & \\
\hline
\end{tabular}

Table 3.

"In-bore" biopsy results stratified according to PIRADS Score.

\begin{tabular}{|lccccc|}
\hline & Overall & $\begin{array}{c}\text { PIRADS score } \\
\mathbf{3}\end{array}$ & $\begin{array}{c}\text { PIRADS score } \\
\text { PIRADS score }\end{array}$ & $\begin{array}{c}\mathbf{p} \\
\text { value }\end{array}$ \\
\hline Number of Pts, (\%) & 39 & $18(46.2)$ & $11(28.2)$ & $10(25.6)$ & - \\
\hline Detection rate PCa, $\mathrm{n}(\%)$ & $18 / 39(53.8)$ & $5 / 18(27.8)$ & $7 / 11(63.6)$ & $6 / 10(60)$ & 0.3 \\
\hline Detection rate csPCa, $\mathrm{n}(\%)$ & $17 / 39(43.5)$ & $3 / 18(16.7)$ & $5 / 11(45.5)$ & $5 / 10(50)$ & 0.1 \\
\hline Detection rate csPCa/PCa, \% & $17 / 18(94.4)$ & $3 / 5(60)$ & $5 / 7(71.4)$ & $5 / 6(83.3)$ & 0.7 \\
\hline ISUP grade group, $\mathrm{n}(\%)$ & & & & & \\
Negative & $21(53.8)$ & $13(72.2)$ & $4(36.4)$ & $4(40)$ & 0.1 \\
1 & $1(2.5)$ & $1(5.5)$ & $0(0)$ & $0(0)$ & \\
2 & $10(25.6)$ & $3(16.6)$ & $5(45.4)$ & $2(20)$ & \\
3 & $4(10.3)$ & $1(5.5)$ & $1(9.1)$ & $2(20)$ & \\
$4-5$ & $3(7.6)$ & $0(0)$ & $1(9.1)$ & $2(20)$ & \\
\hline
\end{tabular}

Table 4.

Number of bioptic cores taken from patients who underwent in-bore and random biopsies.

\begin{tabular}{|lccccc|}
\hline Number of cores & $\begin{array}{c}\text { 0verall } \\
(\mathbf{n}=\mathbf{3 9})\end{array}$ & $\begin{array}{c}\text { Biopsy näive } \\
(\mathbf{n}=\mathbf{3 0})\end{array}$ & $\begin{array}{c}\text { Previous negative } \\
\text { biopsy } \mathbf{( n = 7 )}\end{array}$ & $\begin{array}{c}\text { Active surveillance } \\
(\mathbf{n}=\mathbf{2})\end{array}$ & $\begin{array}{c}\mathbf{p} \\
\text { value }\end{array}$ \\
\hline Total & $13(13-14)$ & $13(13-14)$ & $13(13-15)$ & $13(13-13)$ & 0.5 \\
\hline In bore biopsy & $1(1-2)$ & $1(1-2)$ & $1(1-1)$ & $1(1-1)$ & 0.5 \\
\hline Random biopsy & $12(12-12)$ & $12(12-12)$ & $12(12-14)$ & $12(12-12)$ & 0.5 \\
\hline
\end{tabular}


Table 5.

Overall "detection rates" and "pathologic results" of patients who underwent "in-bore" biopsies and random biopsies.

\begin{tabular}{|lcccc|}
\hline Number of cores & Overall $(\mathbf{n}=\mathbf{3 9})$ & In-bore biopsy & Random biopsy & p value \\
\hline Detection rate PCa, $n(\%)$ & $23 / 39(59)$ & $18 / 39(53.8)$ & $18 / 39(53.8)$ & 0.9 \\
\hline Detection rate csPCa, $n(\%)$ & $20 / 39(51.3)$ & $17 / 39(43.5)$ & $15 / 39(38.4)$ & 0.7 \\
\hline csPCa/PCa detection rate, $\%$ & $20 / 23(87)$ & $17 / 18(94.4)$ & $15 / 18(83.3)$ & 0.8 \\
\hline ISUP grade group, $n(\%)$ & $16(41)$ & $21(53.8)$ & $21(53.8)$ & 0.6 \\
Negative & $4(10.3)$ & $1(2.5)$ & $5(12.8)$ & \\
1 & $11(28.2)$ & $10(25.6)$ & $7(17.9)$ & \\
2 & $6(15.4)$ & $4(10.3)$ & $5(12.8)$ & \\
3 & $2(5.1)$ & $3(7.6)$ & $1(2.6)$ & \\
$4-5$ & & &
\end{tabular}

Table 6.

Overall "detection rates" and "pathologic results" of biopsy näive patients who underwent "in-bore" biopsies and Random biopsies.

\begin{tabular}{|lcccc|}
\hline & Overall $(\mathbf{n}=\mathbf{3 0})$ & In-bore biopsy & Random biopsy & $\mathbf{p}$ value \\
\hline Detection rate PCa, $\mathrm{n}(\%)$ & $16 / 30(75.4 \%)$ & $14 / 30(46.7)$ & $1 / 30(43.3)$ & 0.8 \\
\hline Detection rate csPCa, $\mathrm{n}(\%)$ & $15 / 30(50 \%)$ & $14 / 30(46.6)$ & $13 / 30(43.3)$ & 0.8 \\
\hline csPCa/PCa detection rate, $\%$ & $15 / 16(93.8 \%)$ & $14 / 14(100)$ & $13 / 13(100)$ & 0.5 \\
\hline ISUP grade group, $\mathrm{n}(\%)$ & $14(46.7)$ & $16(53.3)$ & $17(56.7)$ & 0.7 \\
Negative & $1(3.3)$ & $0(0)$ & $0(0)$ & \\
1 & $9(30)$ & $10(33.3)$ & $8(26.7)$ & \\
2 & $5(16.7)$ & $2(6.6)$ & $4(13.3)$ & \\
3 & $1(3.3)$ & $2(6.6)$ & $1(3.3)$ & \\
$4-5$ & & &
\end{tabular}

Table 7.

Overall "detection rates" and "pathologic results" of patients with previous negative biopsies who underwent "in-bore" biopsies and random biopsies.

\begin{tabular}{|lcccc|}
\hline & Overall $(\mathbf{n}=\mathbf{7})$ & In-bore biopsy & Random biopsy & p value \\
\hline Detection rate PCa, $\mathrm{n}(\%)$ & $4 / 7(57.4)$ & $2 / 7(28.6)$ & $2 / 7(28.6)$ & 0.3 \\
\hline Detection rate csPCa, $\mathrm{n}(\%)$ & $2 / 7(42.9)$ & $2 / 7(28.6)$ & $1 / 7(14.2)$ & 0.1 \\
\hline csPCa/PCa detection rate, \% & $2 / 4(50)$ & $2 / 7(28.6)$ & - & - \\
\hline ISUP grade group, $\mathrm{n}(\%)$ & $1(14.3)$ & $5(71.4)$ & $5(71.2)$ & 0.2 \\
Negative & $4(57.1)$ & $0(0)$ & $2(28.6)$ & \\
1 & $0(0)$ & $0(0)$ & $0(0)$ & \\
2 & $1(14.3)$ & $1(14.2)$ & $0(0)$ & \\
3 & $1(14.3)$ & $1(14.2)$ & $0(0)$ & \\
$4-5$ & & &
\end{tabular}

Table 8.

Overall "detection rates" and "pathologic results" of patients in Active Surveillance who underwent "in-bore" biopsies and random biopsies.

\begin{tabular}{|lcccc|}
\hline & Overall $(\mathbf{n}=\mathbf{2})$ & In bore biopsy & Random biopsy & p value \\
\hline Detection rate PCa, $\mathrm{n}(\%)$ & $2 / 2(100$ & $2 / 2(100)$ & $1 / 2(50)$ & 0.5 \\
\hline Detection rate csPCa, $\mathrm{n}(\%)$ & $2 / 2(100)$ & $1 / 2(50)$ & $1 / 2(50)$ & 1 \\
\hline csPCa/PCa detection rate, \% & $2 / 2(100)$ & $1 / 2(50)$ & $1 / 1(100)$ & 0.5 \\
\hline ISUP grade group, $\mathrm{n}(\%)$ & & & & \\
\hline Negative & $0(0)$ & $0(0)$ & $1(50)$ & 0.3 \\
1 & $0(0)$ & $1(50)$ & $0(0)$ & \\
2 & $1(50)$ & $0(0)$ & $1(50)$ & \\
3 & $1(50))$ & $1(50)$ & $0(0)$ & \\
$4-5$ & $0(0)$ & $0(0)$ & $0(0)$ & \\
\hline
\end{tabular}

series. The details of results are reported in table 2-8. As far as the secondary endpoint is concerned, we have not observed any complications related to procedures.

\section{Discussion}

TRUS-GB represents the "gold-standard" technique of histological diagnosis of prostate cancer. Aim of this study was to investigate the real diagnostic accuracy of a novel prostate biopsy technique MRI guided compared to the standard TRUS-guided biopsy (11). During the recent years, there was an increasing use of mpMRI in stadiation of prostate cancer e in order to improve quality of surgery during radical prostatectomy (12).

Other important application of mpMRI is the guide to prostate biopsy in order to improve PCa diagnosis with the aim to refine PCa risk classification (13) and, simultaneously, to overcome the limitations of contemporary standard TRUS-GB: low-risk patients could be spared from biopsies reducing overdetection of low-risk cancer while a lower number of cores could be required in men with suspicious MRI findings reducing also potential complications related to the procedure and all the consequence on quality of life (14-16). Targeted MRI-GB has become an alternative approach to TRUS-GB and several MRI-GB methods have been proposed for the diagnosis of PCa. In the "cognitive" technique, TRUS-GB is planned on the basis of MR images; in spite of reduced costs, this method has a long learning curve and the sampling of suspicious lesions may not be guaranteed. The MRI-US "fusion" biopsy is simply described as a way to align a pre-registered MRI to an intraprocedural US in order to identify and target suspected lesions within the gland through a dedicated hardware platform targeting areas found during mpMRI and not clearly visible during US scan; the advantages are the high reproducibility and the real time feedback though counterbalanced by the high up-front cost of the device. The "in bore" technique consists in the execution of the biopsies directly inside the MRI scanner with dedicate non-magnetic biopsy devices. Despite a longer operative time and higher costs, this method has realtime feedback in needle placement, fewer sampled cores and a low likeli- 
hood of missed target. Therefore, the use of "in bore" MRI-GB has the potential to reduce the sampling error associated with unselective standard biopsy scheme by providing better disease localization; moreover, accurate risk stratification through improved cancer sampling may impact therapeutic decision making $(13,17)$. In our experience the overall detection rate of MRI-GB and TRUS-GB are similar; in particular, in the series of MRIGB, we observed a slightly higher PCa diagnosis of clinically significant disease (43.5\% vs 33.3\%), although this result was not statistically significant. A recent systematic review showed that MRI-GB improved significant PCa detection compared to standard TRUS-GB (relative sensitivity $1.26,95 \%$ CI 1.08-1.46) (18). In their preliminary experience with in-bore technique, Panebianco et al. (19) showed a DR for the diagnosis of PCa of $80 \%$ and $90 \%$ of PCa cases had intermediate grade aggressiveness. Whereas there was currently not enough evidence to recommend mpMRI before a first set of prostate biopsies (2), the use of targeted biopsy often achieved significantly higher cancer DR in the repeat biopsy setting. In patients with prior negative biopsy, MRI-GB showed a median DR of $42 \%$, significantly higher than those reported in repeating systematic biopsy (20). Our preliminary experience in MRI-GB confirmed the feasibility and the reproducibility of an "in-bore" strategy on a 1.5 T MR scanner using a 32-channel coil. Enghelard et al. (21) reported a DR of 38\% in 37 consecutive men with elevated PSA levels and negative prostate biopsies underwent a MRI-GB in a 1.5-T scanner in the supine position; in similar but larger cohorts, Roethke et al. (22) and Hoeks et al. (10) found a DR of 52\% (52/100) and 41\% (14/37), respectively. In our cohort, analyzing the series of patients with previous negative biopsies, the overall DR of MRI-GB was higher than TRUS-GB (57.1\% vs $28.6 \%$ ), particularly in the clinically significant disease $(28.5 \%$ vs $14.2 \%$ ). Our preliminary results highlighted the excellent correlation between the PI-RADS score at mpMRI and the ISUP grade groups in MRI-GB cores: this data allows us to infer, on one hand, a high capability of the mpMRI to predict the biological aggressiveness of neoplastic lesions and, on the other hand, the good performance of the MRI-GB procedure. Moreover, the mpMRI procedure with this setting and patients in the prone position has been well tolerated and feasible to perform. Given an initial learning curve, we believe that the time needed for the procedure can be further reduced optimizing the setting and the positioning of the needle guide. Finally, Jung et al. (23) found that the rate of positive MRI-GB resulted as a function of target size and level of suspicious. It could be argued that obvious that larger target with higher level of suspicious were most likely to yield a positive biopsy; however, the authors concluded that this finding could be helpful when deciding whether to perform a MRI-GB. Engelhard et al. (21) concluded that suspicious lesions with a diameter $>10 \mathrm{~mm}$ could be successfully punctured using this device. In spite of reliable feasibility and the preliminary promising results with an acceptable DR, some limitations of the present study have to be kept in mind. First, the number of patients was small and, as a part of study design, the patients analyzed were only men with posi- tive findings at mpMRI: this selected patient population from a single institution series may have a positive influence on the number of tumors detected; however, the study was thought as an initial experience to compare the MRI-GB technique and standard TRUS-GB in order to evaluate its feasibility. Consequently, the preliminary results are far from being meaningful and have to be interpreted carefully. Second, the study lacks follow up data and no prostate specimen histology can confirm the results of a negative MRI-GB; further series with radical prostatectomy specimen as reference standards are required in order to corroborate the benefit of a MRI-GB procedure. Finally, it is important to highlight that initially MRI-GB can represent a time-consuming and expensive procedure but, as reported by other authors (24), initial costs could be written off by reducing treatment related costs and improving risk tumor evaluation and quality of life preventing unnecessary radical treatment of insignificant tumors.

\section{Conclusions}

MRI-GB represents a promising technique that may offer some of these advantages compared to standard systematic TRUS-GB. Our preliminary experience in MRI-GB resulted safe and feasible and represents a viable procedure for the diagnosis and characterization of PCa especially in a subgroup of patient with clinically significant disease. Further investigations are needed in order to identify who should undergone prostate biopsy and the technique that should be used with the aim of detecting significant PCa and reducing the burden of biopsies.

\section{REFERENCES}

1. Siegel R, Naishadham D, Jemal A. Cancer statistics 2012. CA Cancer J Clin. 2012; 62:10-21.

2. Heidenreich A, Bastian PJ, Bellmunt J, et al. EAU guidelines on prostate cancer, part 1: screening, diagnosis, and local treatment with curative intent - update 2013. Eur Urol. 2014; 65:124-137.

3. Delongchamps NB, De la Roza G, Chandan V, et al. Diagnostic accuracy of extended biopsies for the staging of microfocal prostate cancers in autopsy specimen. Prostate Cancer Prostatic Dis. 2009; 12:137-142.

4. Roehl KA, Antenor JA, Catalona WJ. Serial biopsy results in prostate cancer screening study. J Urol. 2002; 167:2435-9.

5. Jones JS. Saturation biopsy for detecting and characterizing prostate cancer. BJU Int. 2007; 99:1340-1344.

6. Lane BR, Zippe CD, Abouassaly R, et al. Saturation technique does not decrease cancer detection during follow up after initial prostate biopsy. J Urol. 2008; 179:1746-1750.

7. Watanabe Y, Terai A, Araki T, et al. Detection and localization of prostate cancer with the targeted biopsy strategy based on ADC map: a prospective large-scale cohort study. J Magn Reson Imaging. 2012; 35:1414-1421.

8. Numao N, Yoshida S, Komai Y, et al. Usefulness of pre-biopsy multiparametric magnetic resonance imaging and clinical variables to reduce initial prostate biopsy in men with suspected clinically localized prostate cancer. J Urol. 2013; 190:502-508.

10. Siddiqui MM, Rais-Bahrami S, Truong H, et al: Magnetic reso- 
nance imaging/ultrasound fusion biopsy significantly upgrades prostate cancer versus systematic 12-core transrectal ultrasound biopsy. Eur Urol. 2013; 64:713-719.

11. Schiavina R, Vagnoni V, D'Agostino D, et al. "In-bore" MRIguided prostate biopsy using an endorectal nonmagnetic device: a prospective study of 70 consecutive patients. Clin Genitourin Cancer. 2017; 15:417-427.

12. Schiavina R, Bianchi L, Borghesi M, et al. MRI displays the prostatic cancer anatomy and improves the bundles management before robot-assisted radical prostatectomy. J Endourol. 2018; 32:315321.

13. Vagnoni V, Bianchi L, Borghesi M, et al. Adverse features and competing risk mortality in patients with high-risk prostate cancer. Clin Genitourin Cancer. 2017; 15:e239-e248.

14. Porreca A, Noale M, Artibani W, et al. Pros-IT CNR study group. Disease-specific and general health-related quality of life in newly diagnosed prostate cancer patients: the Pros-IT CNR study. Health Qual Life Outcomes. 2018; 16:122.

15. Gacci M, Noale M, Artibani W, et al. Pros-IT CNR study group. Quality of life after prostate cancer diagnosis: data from the Pros-IT CNR. Eur Urol Focus. 2017; 3:321-324.

16. Noale M, Maggi S, Artibani W, et al. Pros-IT CNR study group. Pros-IT CNR: an Italian prostate cancer monitoring project. Aging Clin Exp Res. 2017; 29:165-172.

17. Grasso AA, Cozzi G, De Lorenzis E, et al. Multicenter analysis of pathological outcomes of patients eligible for active surveillance according to PRIAS criteria. Minerva Urol Nefrol. 2016; 68:237-41.
18. Schoots IG, Roobol MJ, Nieboer D. et al. Magnetic resonance targeted biopsy may enhance the diagnostic accuracy of significant prostate cancer detection compared to standard transrectal ultrasound guided biopsy: a systematic review and meta- analysis. Eur Urol. 2015; 68:438-450.

19. Panebianco V, Barchetti F, Manenti G, et al. MR imaging-guided prostate biopsy: technical features and preliminary results. Radiol Med. 2015; 120:571-578.

20. Overduin CG, Futterer JJ, Barentsz JO. MRI-guided biopsy for prostate cancer detection: a systematic review of current clinical results. Curr Urol Rep. 2013; 14:209-213.

21. Engelhard K, Hollenbach HP, Kiefer B, et al. Prostate biopsy in the supine position in a standard 1.5-T scanner under real time MRimaging control using a MR-compatible endorectal biopsy device. Eur Radiol. 2006; 16:1237-1243.

22. Roethke M, Anastasiadis AG, Lichy M, et al. MRI-guided prostate biopsy detects clinically significant cancer: analysis of a cohort of 100 patients after previous negative TRUS biopsy. World J Urol. 2012; 30:213-218.

23. Jung AJ, Westphalen AC, Kurhanewicz J, et al. Clinical Utility of Endorectal MRI-Guided Prostate Biopsy: Preliminary Experience. J Magn Res Imag. 2014; 40:314-323.

24. de Rooij M, Crienen S, Witjes JA, et al. Cost-effectiveness of Magnetic Resonance (MR) Imaging and MR-guided targeted biopsy versus systematic transrectal ultrasound-guided biopsy in diagnosing prostate cancer: a modelling study from a health care perspective. Eur Urol. 2014; 66:430-436.

\section{Correspondence}

Daniele D’Agostino, MD (Corresponding Author)

dott.dagostino@gmail.com

Daniele Romagnoli, MD

dromagnoli@casacura.it

Paolo Corsi, MD

pcorsi@casacura.it

Marco Giampaoli, MD

mgiampaoli@casacura.it

Walter Artibani, MD

prof.artibani@gmail.com

Angelo Porreca, MD

angeloporreca@gmail.com

Department of Robotic Urological Surgery, Abano Terme Hospital

Piazza Cristoforo Colombo 1, 35031 Abano Terme (PD) (Italy)

Federico Mineo Bianchi, MD

federico.mineobianchi@gmail.com

Riccardo Schiavina, MD

rschiavina@yahoo.it

Eugenio Brunocilla, MD

eugenio.brunocilla@unibo.it

Department of Urology, University of Bologna, Bologna (Italy) 